\title{
Heat Transport and Majorana Fermions in a Superconducting Dot-Wire System: An Exact Solution
}

\author{
Oscar Bohórquez and A. M. S. Macêdo \\ Departamento de Física, Laboratório de Física Teórica e Computacional, Universidade Federal de Pernambuco, \\ 50670-901 Recife, Pernambuco, Brazil
}

Correspondence should be addressed to A. M. S. Macêdo; a.murilos.macedo@gmail.com

Received 16 August 2018; Accepted 28 October 2018; Published 2 December 2018

Academic Editor: Andrei D. Mironov

Copyright (C) 2018 Oscar Bohórquez and A. M. S. Macêdo. This is an open access article distributed under the Creative Commons Attribution License, which permits unrestricted use, distribution, and reproduction in any medium, provided the original work is properly cited.

\begin{abstract}
We obtain exact expressions for the first three moments of the heat conductance of a quantum chain that crosses over from a superconducting quantum dot to a superconducting disordered quantum wire. Our analytic solution provides exact detailed descriptions of all crossovers that can be observed in the system as a function of its length, which include ballistic-metallic and metallic-insulating crossovers. The two Bogoliubov-de Gennes (BdG) symmetry classes with time-reversal symmetry are accounted for. The striking effect of total suppression of the insulating regime in systems with broken spin-rotation invariance is observed at large length scales. For a single channel system, this anomalous effect can be interpreted as a signature of the presence of the elusive Majorana fermion in a condensed matter system.
\end{abstract}

\section{Introduction}

Random-matrix theory (RMT) has been widely used in the study of phase-coherent complex quantum systems and has been particularly successful in uncovering universal properties of quantum transport in chaotic and disordered systems [1]. Much of the success of RMT in quantum transport is due to the strong correspondence between the statistical properties of random-matrix ensembles and the fluctuations of measured observables of complex quantum systems as a function of some control parameter, such as energy or magnetic field. The universality of transport properties, such as the moments of the conductance, lies in their independence of microscopic details of the scattering source. Nevertheless, random-matrix ensembles are sensitive to certain intrinsic symmetries of the system, such as time-reversal (TR), spinrotation (SR), electron-hole (e-h), and chiral (Ch). It has been established that these symmetries lead to a classification of RMT ensembles into ten universal classes (the tenfold way) $[2,3]$, which are divided into three categories: (i) WignerDyson (WD, three classes), appropriate to describe normal disordered conductors, (ii) chiral (Ch, three classes), appropriate for systems with a purely off-diagonal disorder, and (iii)
Bogoliubov-de Gennes (BdG, four classes), appropriate for normal-superconducting (NS) hybrid systems.

From the perspective of quantum transport, phasecoherent mesoscopic systems can be classified into two types: (I) disordered conductors, in which impurities generate multiple elastic scatterings with an associated mean free path $l$ that is less than the system's dimensions, and (II) ballistic cavities, where $l$ is greater than the system's dimensions and thus the dominant scattering mechanism is reflection at the border of the cavity. Remarkably, RMT can efficiently describe both types of systems since universal transport properties do not depend on the details of either the impurity potential (for disordered systems) or the shape of the cavity (for ballistic systems) [1]. This insensitivity to microscopic details goes as far as to allow an identification, under certain conditions, of a multichannel disordered quantum wire with a chain of ballistic cavities [4].

Besides RMT, there are other well-developed approaches to quantum transport in both disordered wires and chaotic ballistic cavities: the field-theoretic nonlinear sigma model [5] and the trajectory-based semiclassical approach [6] are the most well known. These three approaches have many advantages and pitfalls, but since they are constructed from 
different and somewhat unrelated statistical hypothesis on the behavior of the underlying degrees of freedom, they may be considered a complementary, albeit equivalent, physical description of the system. Notwithstanding, a full fledged mathematical proof of the equivalence of these three approaches is still missing, in spite of much effort and some successes in particular systems, such as quantum dots with ideal couplings to external leads [7].

In superconducting systems, quantum transport has very striking and different features in comparison with their normal counterpart, which in part is due to what is known as Andreev reflections (AR). The most remarkable phenomenon is probably the possibility of a condensed matter realization of Majorana fermions as protected bound states at the ends of topological superconducting wires [8]. Transport observables in these systems, such as the electrical conductivity, can give information about topological invariants and topological quantum numbers. The thermal conductivity, on the other hand, despite maybe not containing direct information of topological invariants, as can be seen from their randommatrix description [9], can still provide valuable information about topological phase transitions [10]. It was, however, in the study of disordered quantum wires that evidence of the presence of condensed matter Majorana modes emerged most clearly. These can be traced back to the prediction [11] that for quantum wires in the chiral classes (for odd $N$ open scattering channels) and in the superconducting D and DIII classes there is no exponential localization, since, unlike its behavior in the standard classes, the average conductance falls off in the limit of long distances $L \gg N l$ as $1 / \sqrt{L}$, which is a kind of super-ohmic behavior. They also found that in these special classes the average density of states (DOS) diverges logarithmically, $\rho(\varepsilon) \sim 1 /\left|\varepsilon \tau \ln ^{3}(\varepsilon \tau)\right|$, as energy $\varepsilon \longrightarrow 0$ (where $\tau=N^{2} l$ and the Fermi velocity has been set to unity). A similar singularity had already been found by Dyson in the analysis of disordered linear chains [12]. On the other hand, [13] found through a general analysis using strong-disorder renormalization group (RG) that systems of classes $D$ and DIII exhibit localization and an average DOS that vanishes as a power law $\rho(\varepsilon) \sim|\varepsilon \tau|^{\delta-1}$ with $\delta>0$, as $\varepsilon \longrightarrow 0$. Remarkably, at certain critical points obtained by fine-tuning the disorder, both delocalization and Dyson's divergence can be present. The authors of [13] were also among the first to relate this special type of criticality to transitions between topological phases and also to point out that it could be a signature of the existence of Majorana zero modes. Reference [14] confirmed these results and presented evidence that the delocalization at critical points is well described by the DMPK equation of superconductors. Furthermore, they claimed that there may be "superuniversality" combining the chiral class and the superconducting classes D and DIII, since they have certain similar characteristics in regard to these critical points. Later, the authors of [15] argued that the diverging nature of the average density of states at the band center is a signature of topologically protected zero modes bound to point defects.

In this paper we employ random-matrix theory to study two classes of superconducting dot-wire systems. We obtain, in the continuum limit of a quantum chain with TR symmetry, an exact description of the crossover in thermal conduction between a superconducting chaotic ballistic cavity (a quantum dot) and a disordered multichannel superconducting quantum wire. The calculations were guided by a recent classification scheme of RMT Brownian motion ensembles [16] and were performed by means of a multivariate integral transform method proposed in [16, 17]. More specifically, we obtain exact expressions for the first three moments of the heat conductance of two classes of disordered superconducting quantum wires with time-reversal symmetry and with a crossover to a quantum dot in the small length limit. The analytic solution describes in detail various types of crossovers as a function of the systems' length, which include ballistic-metallic and metallic-insulating crossovers. If the system is realized as a single channel topological superconductor with broken spin-rotation invariance, we can interpret the total suppression of the insulating regime as a signature of the presence of a condensed matter Majorana fermion.

\section{The Scattering Problem}

Consider a confined quantum system ideally coupled to two electron reservoirs via point contacts with $N_{1}$ and $N_{2}$ open scattering channels, respectively. According to the LandauerBüttiker scattering formalism [18], coherent particle transfer through such a device can be efficiently described by its scattering matrix, which can be generically written as

$$
S=\left[\begin{array}{ll}
r_{N_{1} \times N_{1}} & t_{N_{1} \times N_{2}} \\
t_{N_{2} \times N_{1}}^{\prime} & r_{N_{2} \times N_{2}}^{\prime}
\end{array}\right],
$$

where $r$ and $r^{\prime}$ are reflection matrices and $t$ and $t^{\prime}$ are transmission matrices. The subscripts denote the matrix dimensions and the matrices $t t^{\dagger}$ and $t^{\prime} t^{\prime \dagger}$ are Hermitian and although they have different spectra, the spectrum of the smaller matrix coincides with the nonzero eigenvalues (transmission eigenvalues) of the bigger one $\left(\tau_{1}, \ldots, \tau_{N}\right) \in$ $[0,1]^{N}$. Transport observables can be conveniently written in terms of these transmission eigenvalues. For instance, the thermal conductance of a superconducting system, at low temperature $T$, is given by [19],

$$
G=G_{0} d \sum_{i}^{N} \tau_{i}
$$

where $G_{0}=\pi^{2} k_{B}^{2} T / 6 h, N=\min \left(N_{1}, N_{2}\right)$, and $d$ is the spin and/or particle-hole degeneracy.

In ballistic chaotic cavities the transmission eigenvalues are strongly correlated random variables, which because of assumption of ergodic dynamics are well described by RMT. According to RMT, the scattering matrix of a ballistic chaotic cavity with ideal contacts is uniformly distributed over its manifold, and thus the probability density is only restricted by the presence or absence of certain symmetries. For the BdG classes the corresponding joint distribution of transmission eigenvalues is given by [19]

$$
P(\{\tau\})=C_{N} \prod_{i<j}\left|\tau_{i}-\tau_{j}\right|^{\beta} \prod_{i} \tau_{i}^{\beta(\mu+1) / 2-1}\left(1-\tau_{i}\right)^{\gamma / 2},
$$

where $\mu=\left|N_{1}-N_{2}\right|$ and $C_{N}$ is a normalization constant. The values of the parameters $\beta$ and $\gamma$ are solely determined 


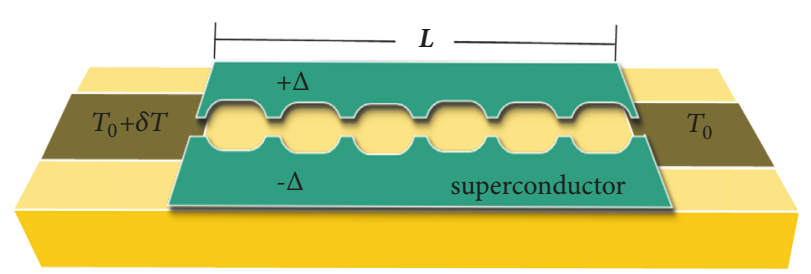

Figure 1: By varying the sample's length $L$, we can go from a quantum dot to a chain of dots, which in the continuous limit is a quantum wire. The conducting device is bounded by superconductors with a phase difference of $\pi$ (order parameters given by $\pm \Delta$ ) and connected to electron reservoirs with different temperatures via ideal contacts.

by the symmetries, as shown in Table II of [19]. Here we shall consider only systems in the presence of TR symmetry, which implies $\beta=2$. Moreover, we must set $\gamma=1$ for systems in the presence of SR symmetry and $\gamma=-1$ for systems with broken SR symmetry. According to the tenfold way of classifying random-matrix ensembles, these classes are denoted by CI and DIII, respectively. We remark that the system described by (3) differs from a normal ballistic cavity, because, in addition to the two normal contacts coupling to the reservoirs, the cavity is geometrically defined by a normal-superconducting interface, which generates Andreev reflections [1]. As a matter of fact, this type of cavity is also known as an Andreev quantum dot [19]. We remark that if we set $\gamma=0$ in (3) we recover the Wigner-Dyson A class for normal systems with broken TR symmetry, which turns out to be a useful way to compare our exact expressions for the moments of the thermal conductance with known results of the literature.

We proceed by combining Andreev quantum dots in a chain geometry, as shown in Figure 1. In such a setup the excitation gap induced by the proximity effect in the inner region is closed by adjusting the superconducting boundaries to have a phase difference of $\pi$, which also ensures that there is no breaking of TR symmetry $[10,19]$. The RMT description of the system can be obtained by appropriately combining the scattering matrices of the Andreev quantum dots, or from the corresponding product of random transfer matrices; see [20] for more details. An equivalent description using the supersymmetric nonlinear sigma model is also possible [21]. Since we want to obtain exact analytical results, we follow $[22,23]$ and take the continuum limit which leads to a Fokker-Planck equation for the evolution, with the sample's length, of the joint probability distribution of transmission eigenvalues with a zero-length initial condition given by the RMT description of an Andreev quantum dot. See [21, 23] for the corresponding problem with normal quantum dots.

The Fokker-Planck equation of a disordered Andreev quantum wire of length $L$, with $N$ open scattering channels and localization length $\xi$, is given by [11]

$$
\frac{\partial P}{\partial s}=\sum_{i}^{N} \frac{\partial}{\partial q_{i}} J \frac{\partial}{\partial q_{i}} \frac{P}{J}
$$

where $s=L /[2 l(\beta N-\beta+\alpha+1)]$ is an adimensional length and

$$
J=\prod_{i<j}\left|\cosh \left(2 q_{i}\right)-\cosh \left(2 q_{j}\right)\right|^{\beta} \prod_{i}^{N} \sinh ^{\alpha}\left(2 q_{i}\right) .
$$

The variables $q_{i}$ are related to the transmission eigenvalues through the relation $\tau_{i}=\operatorname{sech}^{2}\left(q_{i}\right)$ and since we are considering superconducting TR-symmetric systems, we must set $\beta=2$. The parameter $\alpha$ can take the values $\alpha=2$ (in the presence of SR symmetry) and $\alpha=0$ (in the absence of SR symmetry). The WD class is obtained by setting $\alpha=1$ and assuming that TR symmetry is broken.

The problem has thus been reduced to solving (4) with an initial condition given by (3), which is the joint probability distribution of transmission eigenvalues of an Andreev quantum dot. Remarkably, an exact analytical solution can be constructed using an integral transform method [16] which provides a complete description of the crossover of the thermal conductance moments as a function of the system's length, covering all transport regimes: ballistic, metallic, and insulating. For a related study of a normal dot-wire system (WD class) with broken TR symmetry, see [22].

\section{The Integral Transform Method}

A powerful way to represent the probability distributions of the RMT ensembles is to employ a classification scheme based on matrix-valued Brownian motion ensembles [16]. For the BdG classes with TR symmetry (DIII and CI) we may define the following functions:

$$
\begin{aligned}
J_{\beta}(\{x\}) & =\prod_{i<j}^{N}\left|x_{i}-x_{j}\right|^{\beta}, \\
\omega_{N}(\{x\}) & =\prod_{i}^{N} \omega\left(x_{i}\right),
\end{aligned}
$$

where the random variables $x_{i}$ are related to the transmission eigenvalues $\tau_{i}$ via a simple procedure described in [16]. For a ballistic cavity we may follow [24] and take $x_{i}=\tau_{i}$ and write the joint distribution as the stationary solution of the corresponding Brownian motion. We thus get

$$
P^{(0)}(\{x\})=C_{N} J_{\beta}(\{x\}) \omega_{N}(\{x\}),
$$

where the function $\omega(x)$ is given in Table 1. Equation (7) will be used as the initial condition of our crossover problem. On the other hand, for a quasi-one-dimensional quantum wire we may follow [25] and take $x_{i}=\cosh \left(2 q_{i}\right)=\left(2-\tau_{i}\right) / \tau_{i}$ and write the corresponding Fokker-Planck equation as

$$
\frac{\partial P}{\partial s}=\sum_{i}^{N} \frac{\partial}{\partial x_{i}} s\left(x_{i}\right) \omega_{N} J_{\beta} \frac{\partial}{\partial x_{i}} \frac{P}{\omega_{N} J_{\beta}},
$$

where the functions $\omega(x)$ and $s(x)$ are also given in Table 1.

Instead of directly solving Fokker-Plank equation (8) with initial condition (7), we employ an integral transform 
TABLE 1: Classification of Brownian motion ensembles for the BdG class.

\begin{tabular}{lcccc}
\hline RMT & random variable & $\omega(x)$ & $s(x)$ & $\mathrm{a}$ \\
\hline $\operatorname{dot}$ & $x_{i}=\tau_{i}$ & $x^{\beta(\mu+1) / 2-1}(1-x)^{\gamma / 2}$ & $x(1-x)$ & 0 \\
wire & $x_{i}=\frac{2}{\tau_{i}}-1$ & $\left(x^{2}-1\right)^{(\alpha-1) / 2}$ & $x^{2}-1$ & 1 \\
& & & $\infty$ \\
\hline
\end{tabular}

method that effectively maps (8) onto a much simpler FokkerPlanck problem in an image space of smaller dimension. The multidimensional integral transform is defined as [16]

$$
W(\{\vartheta\}, s)=\int d^{N} x \Omega_{\beta}(\{x\},\{\vartheta\}) P(\{x\}, s),
$$

where the kernel $\Omega_{\beta}$ is chosen to have only two $\{\vartheta\}$ variables, which is the minimal number of image variables that allows the exact calculation of the first three heat conductance moments. We remark that the integral transform cannot be inverted in general and thus our method cannot produce the full joint distribution of the transmission eigenvalues $P(\{x\}, s)$. However, in [16] a more general choice of the kernel $\Omega_{\beta}$ is presented and from it one could in principle calculate all moments of the conductance. We proceed by defining

$$
\Omega_{\beta}(\{x\},\{\vartheta\})=\prod_{i}^{N} \frac{x_{i}-\vartheta_{0,1}}{x_{i}-\vartheta_{1,1}} .
$$

The Fokker-Planck equation in image space $\{\vartheta\}$ is then given by

$$
\left(\frac{\partial}{\partial s}-\mathscr{M}_{\vartheta}^{\dagger}\right) W(\{\vartheta\}, s)=0
$$

where

$$
\mathscr{M}_{\vartheta}^{\dagger}=\frac{1}{V B} \sum_{i=0}^{1}(-1)^{1+i} \frac{\partial}{\partial \vartheta_{i, 1}}\left(s\left(\vartheta_{i, 1}\right) V B \frac{\partial}{\partial \vartheta_{i, 1}}\right)
$$

and we defined

$$
\begin{aligned}
& V=\frac{\omega\left(\vartheta_{0,1}\right)}{\omega\left(\vartheta_{1,1}\right)}, \\
& B=\frac{1}{\left(\vartheta_{0,1}-\vartheta_{1,1}\right)^{2}} .
\end{aligned}
$$

We can now perform the transformation

$$
W(\{\vartheta\}, s)=1+\omega\left(\vartheta_{1,1}\right) B^{-1 / 2} \Psi(\{\vartheta\}, s)
$$

that maps (11) onto a Schrödinger equation in imaginary time $(s \longrightarrow$ it); thus

$$
\frac{\partial \Psi}{\partial t}+\mathscr{H} \Psi=0
$$

where

$$
\mathscr{H}=\sum_{i=0}^{1}(-1)^{i} \frac{1}{\omega\left(\vartheta_{i}\right)} \frac{\partial}{\partial \vartheta_{i}}\left(\omega\left(\vartheta_{i}\right) s\left(\vartheta_{i}\right) \frac{\partial}{\partial \vartheta_{i}}\right)
$$

is a free particle Hamiltonian in image space.
We proceed by calculating $W(\{\vartheta\}, 0)$ using the joint distribution of the Andreev quantum dot (7). Here we may again use the integral transform method with the following modified kernel:

$$
\Omega_{\beta}^{(0)}(\{\tau\},\{\vartheta\})=\left(\frac{1-\vartheta_{0}}{1-\vartheta_{1}}\right)^{N} \prod_{i}^{N} \frac{\tau_{i}-2 /\left(1-\vartheta_{0}\right)}{\tau_{i}-2 /\left(1-\vartheta_{1}\right)},
$$

where $\tau_{i}=x_{i}$. The choice of kernel is motivated from the connection between the supersymmetric nonlinear sigma model and RMT [22]. From the stationary solution of the corresponding Fokker-Planck equation, we find (Appendix A)

$$
\begin{aligned}
& W(\{\vartheta\}, 0)=1+\left(\vartheta_{0}-\vartheta_{1}\right) \\
& \quad \cdot \sum_{l=0}^{N-1} \frac{\left(1-\vartheta_{0}\right)^{l}}{\left(1-\vartheta_{1}\right)^{l+1}}\left(f_{N-l-1}\left(\vartheta_{0}\right) g_{N-l-1}\left(\vartheta_{1}\right)-1\right),
\end{aligned}
$$

where

$$
\begin{aligned}
& f_{n}\left(\vartheta_{0}\right)=F\left[-n,-n-\mu ;-2 n-\mu-\frac{\gamma}{2} ; \frac{1-\vartheta_{0}}{2}\right], \\
& g_{n}\left(\vartheta_{1}\right) \\
& \quad=F\left[n+1, n+1+\mu ; 2 n+\mu+\frac{\gamma}{2}+2 ; \frac{1-\vartheta_{1}}{2}\right],
\end{aligned}
$$

and $F[a, b ; c ; z]$ denotes the hypergeometric function.

We are now in position to unify the notations for the symmetry indices $\gamma$ and $\alpha$ of the quantum dot and the quantum wire, respectively. For that, we introduce the new index

$$
\nu=\frac{\alpha-1}{2}=\frac{\gamma}{2}
$$

which can have two values: $v=1 / 2$ (system with TR and SR symmetry) or $v=-1 / 2$ (systems with TR symmetry and no SR symmetry). In the tenfold way of $S$-matrix classification, these classes correspond to CI and DIII, respectively. We remark that $v=0$ corresponds to the Wigner-Dyson A class (systems with no TR symmetry).

\section{Exact Solution for the Dot-Wire System}

We can now address the full problem and solve (11), which describes a multichannel superconducting quantum wire, with a quantum dot initial condition given by (18). It will prove convenient to use kernel (10) with the choices $\vartheta_{0,1}=$ $-\vartheta_{0}$ and $\vartheta_{1,1}=-\vartheta_{1}$, so that we may write

$$
\Omega_{\beta}(\{x\},\{\vartheta\})=\prod_{i}^{N} \frac{x_{i}+\vartheta_{0}}{x_{i}+\vartheta_{1}} .
$$


Note that after using the relation $x_{i}=2 / \tau_{i}-1$ we recover (17), as expected.

Following the procedure introduced in $[16,22]$ we can find the eigenvalues of the Hamiltonian $\mathscr{H}$ shown in (16). We start by specifying the domains of the variables $\vartheta_{0}$ and $\vartheta_{1}$. Inspired by the supersymmetry calculations of [22], we set $-1 \leq \vartheta_{0} \leq 1$ and $1 \leq \vartheta_{0} \leq \infty$. In this domain the eigenvalues of $\mathscr{H}$ are $\varepsilon_{n k}=k^{2}+(n+v+1 / 2)^{2}$ and the corresponding eigenfunctions are (Appendix B)

$$
\varphi_{n k}\left(\vartheta_{0}, \vartheta_{1}\right)=\frac{A_{k}^{(-\nu)}}{\left(h_{n}^{(\nu)}\right)^{1 / 2}} P_{n}^{(\nu)}\left(\vartheta_{0}\right) \frac{F_{k}^{(-\nu)}\left(\vartheta_{1}\right)}{\omega\left(\vartheta_{1}\right)},
$$

where $h_{n}^{(\nu)} \equiv h_{n}^{(\nu, \nu)}$ and $A_{k}^{(\nu)}$ are appropriate normalization constants of the Jacobi polynomials $P_{n}^{(\nu)}\left(\mathcal{V}_{0}\right) \equiv P_{n}^{(\nu, \nu)}\left(\mathcal{V}_{0}\right)$ and the hypergeometric function

$$
F_{k}^{(v)}\left(\vartheta_{1}\right)=F\left[v+\frac{1}{2}+i k, v+\frac{1}{2}-i k ; v+1 ; \frac{1-\vartheta_{1}}{2}\right],
$$

respectively (see Appendices A and B).

We can now construct Green's function of the problem by means of its spectral resolution in terms of the eigenfunctions and the eigenvalues of $\mathscr{H}$. We find

$$
\begin{aligned}
& G\left(\{\vartheta\},\left\{\vartheta^{\prime}\right\}, t\right)=\left(1-\vartheta_{0}^{\prime 2}\right)^{\nu}\left(\vartheta_{1}^{\prime 2}-1\right)^{\nu} \\
& \cdot \sum_{n=0}^{\infty} \int_{0}^{\infty} d k \varphi_{n k}\left(\vartheta_{0}, \vartheta_{1}\right) \varphi_{n k}\left(\vartheta_{0}^{\prime}, \vartheta_{1}^{\prime}\right) e^{-i \varepsilon_{n k} t} .
\end{aligned}
$$

From the completeness of the eigenfunctions, it follows immediately that

$$
G\left(\{\vartheta\},\left\{\mathfrak{\vartheta}^{\prime}\right\}, 0\right)=\delta\left(\vartheta_{0}-\vartheta_{0}^{\prime}\right) \delta\left(\vartheta_{1}-\vartheta_{1}^{\prime}\right),
$$

as expected for a propagator. Using (24) we can write (14) as

$$
\begin{gathered}
W(\{\vartheta\}, s)=1+\left(\vartheta_{0}-\vartheta_{1}\right) \omega\left(\vartheta_{1}\right) \int_{-1}^{1} d \vartheta_{0}^{\prime} \\
\cdot \int_{1}^{\infty} d \vartheta_{1}^{\prime} G\left(\{\vartheta\},\left\{\vartheta^{\prime}\right\}, s\right) \frac{W\left(\vartheta^{\prime}, 0\right)-1}{\left(\vartheta_{0}^{\prime}-\vartheta_{1}^{\prime}\right) \omega\left(\vartheta_{1}^{\prime}\right)} .
\end{gathered}
$$

Inserting (18) and (24) into (26), we get

$$
\begin{aligned}
& W(\{\vartheta\}, s)=1+\left(\vartheta_{0}-\vartheta_{1}\right) \\
& \quad \cdot \sum_{n=0}^{\infty} \int_{0}^{\infty} d k \frac{\left(A_{k}^{(-\nu)}\right)^{2}}{h_{n}^{(\nu)}} e^{-\varepsilon_{n k} s} P_{n}^{(\nu)}\left(\vartheta_{0}\right) F_{k}^{(-\nu)}\left(\vartheta_{1}\right) \\
& \quad \times \sum_{l=0}^{N-1}\left(I_{n l}^{(1)} J_{k l}^{(1)}-I_{n l}^{(0)} J_{k l}^{(0)}\right),
\end{aligned}
$$

where $I_{n l}^{(i)}$ and $J_{k l}^{(i)}, i \in\{0,1\}$, are obtained from the following integrals:

$$
\begin{aligned}
I_{n l}^{(0)} & =\int_{-1}^{1} d \vartheta_{0}\left(1-\vartheta_{0}^{2}\right)^{v} P_{n}^{(v)}\left(\vartheta_{0}\right)\left(1-\vartheta_{0}\right)^{l}, \\
J_{k l}^{(0)} & =\int_{1}^{\infty} d \vartheta_{1} \frac{F_{k}^{(-v)}\left(\vartheta_{1}\right)}{\left(\vartheta_{1}^{2}-1\right)^{v}\left(1-\vartheta_{1}\right)^{l+1}}, \\
I_{n l}^{(1)} & =\int_{-1}^{1} d \vartheta_{0}\left(1-\vartheta_{0}^{2}\right)^{v} P_{n}^{(v)}\left(\vartheta_{0}\right)\left(1-\vartheta_{0}\right)^{l} f_{N-l-1}\left(\vartheta_{0}\right), \\
J_{k l}^{(1)} & =\int_{1}^{\infty} d \vartheta_{1} \frac{F_{k}^{(-v)}\left(\vartheta_{1}\right)}{\left(\vartheta_{1}^{2}-1\right)^{v}\left(1-\vartheta_{1}\right)^{l+1}} g_{N-l-1}\left(\vartheta_{1}\right) .
\end{aligned}
$$

The integrals are calculated in Appendix C for arbitrary $N_{1}$ and $\mathrm{N}_{2}$. The final result is summarized in the following theorem.

Theorem 1. The solution of Fokker-Planck equation (11) with an initial condition given by (18), in the space of the coordinates $\{v\}$, is given by

$$
\begin{gathered}
W(\{\vartheta\}, s)=1+2\left(\vartheta_{0}-\vartheta_{1}\right) \sum_{n=0}^{N-1} \frac{P_{n}^{(\nu)}\left(\vartheta_{0}\right) P_{n}^{(\nu)}(1)}{h_{n}^{(\nu)}} \\
\cdot \int_{0}^{\infty} d \mu_{n k} c_{n k}^{(\nu)}\left(N_{1}\right) c_{n k}^{(\nu)}\left(N_{2}\right) F_{k}^{(-\nu)}\left(\vartheta_{1}\right) e^{-\varepsilon_{n k} s},
\end{gathered}
$$

where

$$
d \mu_{n k}=d k \frac{|\Gamma(1 / 2-v+i k)|^{2}}{|\Gamma(i k)|^{2} \varepsilon_{n k}}
$$

and

$$
c_{n k}^{(v)}(N)=\frac{|\Gamma(N+v+1 / 2+i k)|^{2}}{(N-n-1) ! \Gamma(N+n+2 v+1)} .
$$

This theorem is the central result of this work. It can be used as a generating function to find the first three moments of the thermal conductance. A noteworthy feature of the exact result is the separation of the left-right boundary conditions in the form of the product $c_{n k}^{(v)}\left(N_{1}\right) c_{n k}^{(v)}\left(N_{2}\right)$, which as we will see later allows a simple identification of different conducting regimes.

\section{Application: Moments of the Heat Conductance}

As an application of (29) we calculate the first three moments of the heat conductance. The Landauer formula for the dimensionless thermal conductance is

$$
g=\sum_{i}^{N} \tau_{i}=\sum_{i}^{N} \frac{2}{1+x_{i}} .
$$

From (9), (21), and (32) it is straightforward to verify that the first three moments of thermal conduction can be obtained from the generating function as 

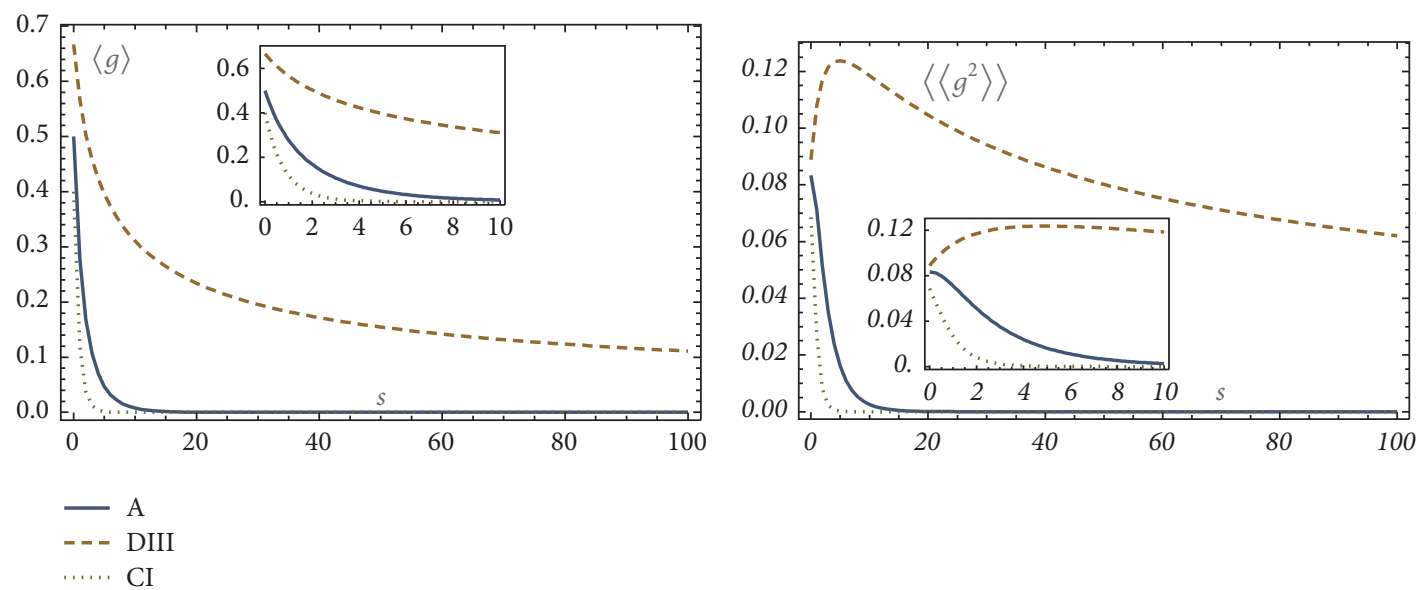

(a)

(b)

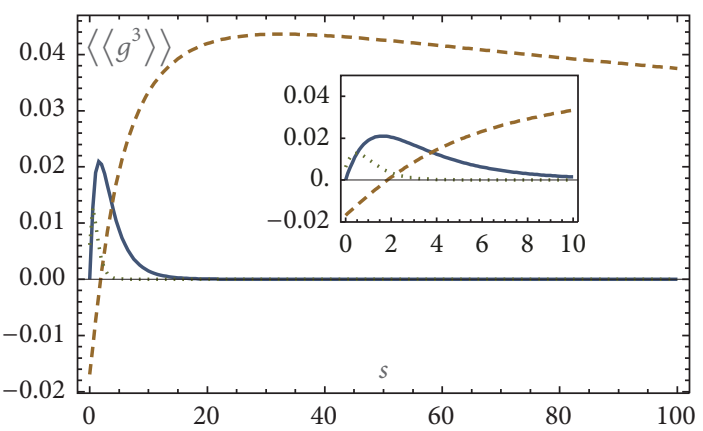

(c)

Figure 2: The first three cumulants of the thermal conductance for the WD (full line) and BdG classes DIII (dashed line) and CI (dotted line) with a single open channel: $N_{1}=N_{2}=1$. The insets show the small $s$ behaviors. Note the qualitative difference of the class DIII BdG system.

$$
\begin{aligned}
\langle g\rangle & =\left.\frac{2 \partial W}{\partial \vartheta_{0}}\right|_{\vartheta_{0}=1=\vartheta_{1}} \\
\left\langle g^{2}\right\rangle & =-\left.\frac{4 \partial^{2} W}{\partial \vartheta_{0} \partial \vartheta_{1}}\right|_{\vartheta_{0}=1=\vartheta_{1}}, \\
\left\langle g^{3}\right\rangle & =\left.4\left(\frac{\partial^{3} W}{\partial \vartheta_{0} \partial \vartheta_{1}^{2}}-\frac{\partial^{3} W}{\partial \vartheta_{1} \partial \vartheta_{0}^{2}}\right)\right|_{\vartheta_{0}=1=\vartheta_{1}} .
\end{aligned}
$$

From (29) we get

$$
\begin{aligned}
\left\langle g^{m}\right\rangle= & 4 \sum_{n=0}^{N-1} \frac{\left(P_{n}^{(v)}(1)\right)^{2}}{h_{n}^{(v)}} \\
& \cdot \int_{0}^{\infty} d \mu_{n k} g_{n k}^{(m)} c_{n k}^{(v)}\left(N_{1}\right) c_{n k}^{(v)}\left(N_{2}\right) e^{-\varepsilon_{n k} s},
\end{aligned}
$$

where

$$
\begin{aligned}
& g_{n k}^{(1)}=1, \\
& g_{n k}^{(2)}=\frac{k^{2}+(1 / 2-v)^{2}}{(1-v)}+\frac{n(n+2 v+1)}{(1+v)}, \\
& g_{n k}^{(3)}=\frac{\left(k^{2}+(1 / 2-v)^{2}\right)\left(k^{2}+(3 / 2-v)^{2}\right)}{2(2-v)(1-v)}
\end{aligned}
$$

$$
\begin{aligned}
& +\frac{2 n(n+2 v+1)\left(k^{2}+(1 / 2-v)^{2}\right)}{(1+v)(1-v)} \\
& +\frac{n(n-1)(n+2 v+1)(n+2 v+2)}{2(1+v)(2+v)} .
\end{aligned}
$$

Equation (34) is a very general exact result. It is valid for any number of channels $N_{1}$ and $N_{2}$ and for any value of the dimensionless length of the quantum wire $s$. It is the central application of this paper and as it stands it can serve as a useful tool to compare with results from other nonperturbative approaches, such as the trajectory-based semiclassical technique and field-theoretic methods.

In Figure 2 we show the behavior of the first three cumulants (denoted with double brackets) of the thermal conductance, as a function of the system's length, of both the WD (full line) and BdG classes DIII (dashed line) and CI (dotted line) for the case of a single channel in each lead. This case is particularly interesting, since as shown in [19] class DIII can be realized using Majorana modes of topological superconductors. Therefore, the unusual shape of the heat conductance moments in this class can be interpreted as a signature of the presence of Majorana fermions in the system. 
In the long length limit, $s \gg 1$, we may use the saddle-point method in (34) to obtain more explicit analytic expressions for each class. For class A, we get

$$
\begin{aligned}
\langle g\rangle & =4\left\langle g^{2}\right\rangle=\frac{64}{9}\left\langle g^{3}\right\rangle \\
& =2 c_{00}^{(0)}\left(N_{1}\right) c_{00}^{(0)}\left(N_{2}\right)\left(\frac{\pi}{s}\right)^{3 / 2} e^{-s / 4},
\end{aligned}
$$

whilst for classes DIII and CI we find, respectively,

$$
\begin{aligned}
\langle g\rangle & =\frac{3}{2}\left\langle g^{2}\right\rangle=\frac{15}{8}\left\langle g^{3}\right\rangle=\frac{2}{\sqrt{\pi s}}, \\
\langle g\rangle & =s\left\langle g^{2}\right\rangle=3 s\left\langle g^{3}\right\rangle \\
& =4 c_{00}^{(1 / 2)}\left(N_{1}\right) c_{00}^{(1 / 2)}\left(N_{2}\right) \frac{e^{-s}}{\sqrt{\pi s}} .
\end{aligned}
$$

From these equations, we see that the BdG DIII class shows anomalous power law behaviors in all three moments. In contrast, the other two classes (WD and CI) show a rapid exponential decay of the moments as a function of $s$, which is a common signature of Anderson localization. The significant attenuation in the decay of the moments for the DIII class as a function of the system's length is thus a kind of delocalization effect, which can be interpreted as indicating a type of anomalous metallic behavior. Since a normal disordered quantum wire shows exponential localization when its length exceeds the localization length, we must conclude that the localization length diverges for a class DIII BdG system. A similar conclusion has been reached in [26] for a thick quantum wire in series with a quantum point contact, which can be described by a DMPK Fokker-Planck equation with a delta function initial condition.

Another noteworthy feature comes from the fact that $c_{n k}^{(v)}(N)=1$ when $N \longrightarrow \infty$. Using this property we can recover several previous results of the literature. The most interesting cases are the following: (a) thick wire: we take $N_{1} \longrightarrow \infty$ and $N_{2} \longrightarrow \infty$, which reproduces the results found in [27], and (b) the disordered quantum wire limit: we take $N_{1} \longrightarrow \infty$ with $N_{2}$ fixed or $N_{2} \longrightarrow \infty$ with $N_{1}$ fixed, which reproduces the results shown in [26].

It is also useful to investigate the system's behavior close to the ballistic limit, i.e., when $s \ll 1 / g_{c}$, where $g_{c}=$ $N_{1} N_{2} /\left(N_{1}+N_{2}\right)$. For that, we expand (34) around $s=0$ to obtain

$$
\begin{aligned}
\langle g\rangle & =\frac{N_{1} N_{2}}{N_{1}+N_{2}+v}(1 \\
- & \left.\frac{\left(N_{1}+v\right)\left(N_{2}+v\right)\left(N_{1}+N_{2}+2 \nu\right)}{\left(N_{1}+N_{2}+v\right)^{2}-1} s+\cdots\right)
\end{aligned}
$$

and

$$
\left\langle\left\langle g^{2}\right\rangle\right\rangle=\frac{N_{1}\left(N_{1}+v\right) N_{2}\left(N_{2}+v\right)}{\left(N_{1}+N_{2}+v\right)^{2}\left(\left(N_{1}+N_{2}+v\right)^{2}-1\right)}(1
$$

$$
\left.+\frac{2\left(\left(N_{1}-N_{2}\right)^{2}-v^{2}\right)\left(N_{1}+N_{2}+2 \nu\right)}{\left(N_{1}+N_{2}+\nu\right)^{2}-4} s+\cdots\right) \text {. }
$$

Note that the zero-order terms reproduce the results of the quantum dot for the three classes [24], as expected. The correction terms are new predictions that may be useful for comparison with alternative techniques, such as the trajectory-based semiclassical approach.

Finally, in Figure 3 we show the behavior of the first three cumulants of the DIII class as a function of the number of channels in each lead. We keep $N_{1}=N_{2}$ and compare the result with the thick-wire limit $N \longrightarrow \infty$. We observe that said limit is reached quickly after an increase of a few channels. The same is also true for the WD and CI classes.

\section{Summary and Conclusions}

We employed random-matrix theory and matrix-valued Brownian motion models to study two classes of superconducting quantum chains. In the continuum dot-wire limit, we find an exact description of the crossover in thermal conduction between a superconducting chaotic ballistic cavity (a quantum dot) and a disordered multichannel superconducting quantum wire. We obtained exact expressions for the first three moments of the heat conductance of two classes of superconducting dot-wire systems with time-reversal symmetry. The analytic solution describes in detail various types of crossovers as a function of the systems' length, which include ballistic-metallic and metallic-insulating crossovers. Interestingly, in the single channel case, if the system is realized experimentally as a topological superconductor, we can interpret the total suppression of the insulating regime in class DIII as a signature of the presence of condensed matter Majorana fermions.

\section{Appendix}

\section{A.}

The generating function of the Andreev quantum dot can be calculated by means of (16) and the kernel shown in (17). Using the variables $\eta_{0}=\left(3+v_{o}\right) /\left(1-v_{o}\right)$ and $\eta_{1}=\left(3+v_{1}\right) /(1-$ $\left.v_{1}\right)$, we find the effective Hamiltonian

$$
\begin{aligned}
\mathscr{H}= & \left(1-\eta_{0}^{2}\right) \frac{\partial^{2}}{\partial \eta_{0}^{2}}+\left(\mu-\frac{\gamma}{2}-\left(2+\mu+\frac{\gamma}{2}\right) \eta_{0}\right) \frac{\partial}{\partial \eta_{0}} \\
& -\left(1-\eta_{1}^{2}\right) \frac{\partial^{2}}{\partial \eta_{1}^{2}} \\
& -\left(\mu-\frac{\gamma}{2}-\left(2+\mu+\frac{\gamma}{2}\right) \eta_{1}\right) \frac{\partial}{\partial \eta_{1}} .
\end{aligned}
$$

The stationary solution of the corresponding Fokker-Planck equation can be written in terms of the eigenfunctions of (A.1), which are Jacobi polynomials $P_{n}^{(a, b)}$ and Jacobi 


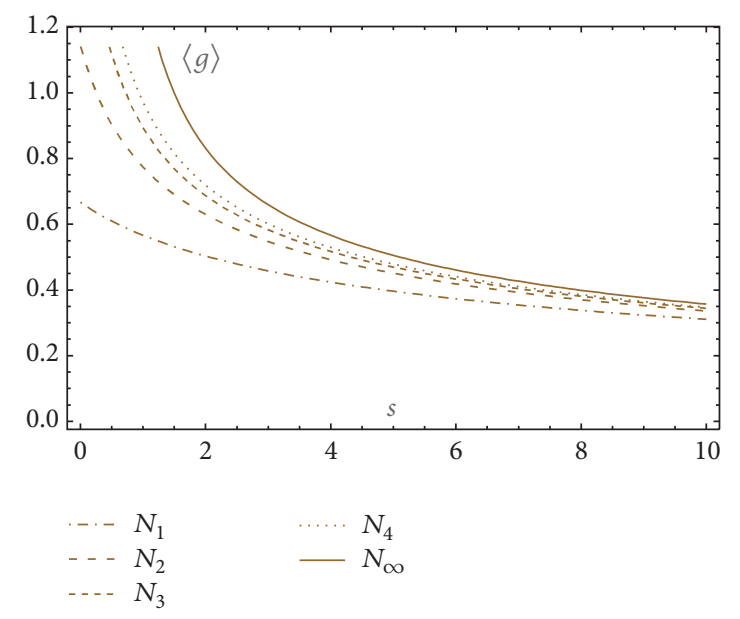

(a)

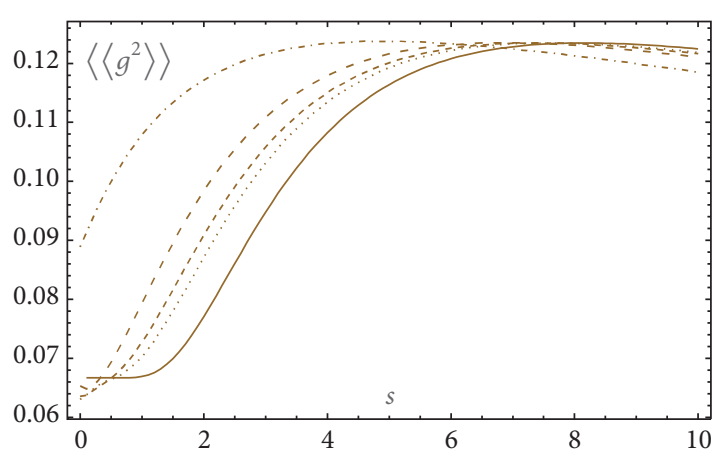

(b)

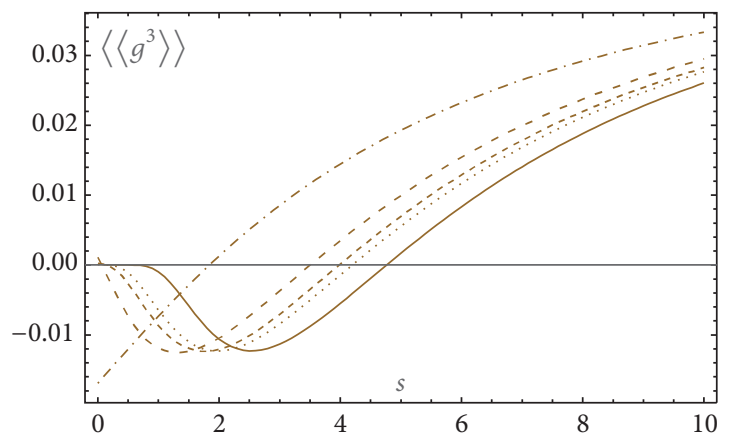

(c)

Figure 3: Variation of the first three cumulants of the thermal conductance for the DIII class with different numbers of channels $N$. $N_{1}=$ $N_{2}=N$.

functions of the second type $Q_{n}^{(a, b)}$. Inserting this result into (14) we get

$$
\begin{aligned}
& W(\{\vartheta\}, 0)=\left(\frac{1-\nu_{o}}{1-\nu_{1}}\right)^{N} \\
& \quad\left(1+2^{3+2 \mu+\gamma / 2} \frac{\left(\nu_{0}-\nu_{1}\right)\left(1+\nu_{1}\right)^{\gamma / 2}}{\left(1-\nu_{0}\right)\left(1-\nu_{1}\right)^{\mu+\gamma / 2+1}} R\right),
\end{aligned}
$$

where

$$
R=\sum_{n=0}^{N-1} \frac{1}{h_{n}^{(\gamma / 2, \mu)}} P_{n}^{(\gamma / 2, \mu)}\left(\frac{3+\nu_{o}}{1-\nu_{o}}\right) Q_{n}^{(\gamma / 2, \mu)}\left(\frac{3+\nu_{1}}{1-\nu_{1}}\right)
$$

and

$$
h_{n}^{(\alpha, \beta)}=\frac{2^{\alpha+\beta+1} \Gamma(n+\alpha+1) \Gamma(n+\beta+1)}{n !(2 n+\alpha+\beta+1) \Gamma(n+\alpha+\beta+1)},
$$

which after some simple algebraic manipulations yields (18).
B.

The generating function of the Andreev quantum wire can be calculated by using (16), which can be written as

$$
\begin{aligned}
\mathscr{H}= & \left(\vartheta_{0}^{2}-1\right) \frac{\partial^{2}}{\partial \vartheta_{0}^{2}}+2(\nu+1) \vartheta_{0} \frac{\partial}{\partial \vartheta_{0}}-\left(\vartheta_{1}^{2}-1\right) \frac{\partial^{2}}{\partial \vartheta_{1}^{2}} \\
& -2(\nu+1) \vartheta_{1} \frac{\partial}{\partial \vartheta_{1}} .
\end{aligned}
$$

Note that the first two terms of this equation match with the operator of the Jacobi differential equation with equal parameters $P_{n}^{(\nu)}\left(\vartheta_{0}\right) \equiv P_{n}^{(\nu, \nu)}\left(\vartheta_{0}\right)$

$$
\begin{aligned}
& {\left[\left(\vartheta_{0}^{2}-1\right) \frac{\partial^{2}}{\partial \vartheta_{0}^{2}}+2(\nu+1) \vartheta_{0} \frac{\partial}{\partial \vartheta_{0}}\right] P_{n}^{(v)}\left(\vartheta_{0}\right)} \\
& \quad=n(n+2 v+1) P_{n}^{(v)}\left(\vartheta_{0}\right) .
\end{aligned}
$$

On the other hand, the last two terms of (B.1) match with the operator of hypergeometric differential equation (23) for $F_{k}^{(v)}\left(\vartheta_{1}\right)$ 


$$
\begin{aligned}
& {\left[-\left(\vartheta_{1}^{2}-1\right) \frac{\partial^{2}}{\partial \vartheta_{1}^{2}}-2(\nu+1) \vartheta_{1} \frac{\partial}{\partial \vartheta_{1}}\right] F_{k}^{(v)}\left(\vartheta_{1}\right)} \\
& =\left(k^{2}+\nu(\nu+1)+\frac{1}{4}\right) F_{k}^{(v)}\left(\vartheta_{1}\right) .
\end{aligned}
$$

The orthogonality and completeness relations are, respectively,

$$
\begin{aligned}
& \int_{-1}^{1} d \vartheta_{0}\left(1-\vartheta_{0}^{2}\right)^{\nu} P_{n}^{(\nu)}\left(\vartheta_{0}\right) P_{n^{\prime}}^{(\nu)}\left(\vartheta_{0}\right)=\delta_{n n^{\prime}} h_{n}^{(\nu)} \\
& \sum_{n=0}^{\infty} \frac{P_{n}^{(v)}\left(\vartheta_{0}\right) P_{n}^{(v)}\left(\vartheta_{0}^{\prime}\right)}{h_{n}^{(v)}}=\frac{\delta\left(\vartheta_{0}-\vartheta_{0}^{\prime}\right)}{\left(1-\vartheta_{0}^{2}\right)^{\nu}}
\end{aligned}
$$

and

$$
\begin{aligned}
\int_{1}^{\infty} d \vartheta_{1}\left(\vartheta_{1}^{2}-1\right)^{\nu} F_{k}^{(v)}\left(\vartheta_{1}\right) F_{k^{\prime}}^{(v)}\left(\vartheta_{1}\right) & =\frac{\delta\left(k-k^{\prime}\right)}{\left(A_{k}^{(v)}\right)^{2}} \\
\int_{0}^{\infty} d k\left(A_{k}^{(v)}\right)^{2} F_{k}^{(v)}\left(\vartheta_{1}\right) F_{k}^{(v)}\left(\vartheta_{1}^{\prime}\right) & =\frac{\delta\left(\vartheta_{1}-\vartheta_{1}^{\prime}\right)}{\left(\vartheta_{1}^{2}-1\right)^{\nu}}
\end{aligned}
$$

where

$$
\left(A_{k}^{(\nu)}\right)^{2}=\frac{|\Gamma(\nu+1 / 2+i k)|^{2}}{2^{2 \nu}(\Gamma(\nu+1))^{2}|\Gamma(i k)|^{2}} .
$$

Then the eigenfunctions and the eigenvalues of $\mathscr{H}$ are given by

$$
\begin{aligned}
\varphi_{n k}\left(\vartheta_{0}, \vartheta_{1}\right) & =\frac{A_{k}^{(v)}}{\left(h_{n}^{(v)}\right)^{1 / 2}} P_{n}^{(v)}\left(\vartheta_{0}\right) F_{k}^{(v)}\left(\vartheta_{1}\right), \\
\varepsilon_{n k} & =k^{2}+\left(n+v+\frac{1}{2}\right)^{2}
\end{aligned}
$$

with $-1 \leq \vartheta_{0} \leq 1$ and $1 \leq \vartheta_{0} \leq \infty$. For the calculations of the moments of the heat conductance it is convenient to replace $F_{k}^{(\nu)}\left(\vartheta_{1}\right)$ by the following function in (B.3):

$$
\begin{aligned}
\widetilde{F}_{k}^{(v)}\left(\vartheta_{1}\right) & \equiv \frac{1}{\omega\left(\vartheta_{1}\right)} \int_{0}^{\infty} d x \frac{\omega(x) F_{k}^{(v)}(x)}{x+\vartheta_{1}} \\
& =\frac{1}{\omega\left(\vartheta_{1}\right)}\left|\Gamma\left(\frac{1}{2}-v+i k\right)\right|^{2} F_{k}^{(-v)}\left(\vartheta_{1}\right),
\end{aligned}
$$

so the new set of eigenfunctions $\varphi_{n k}\left(\vartheta_{0}, \vartheta_{1}\right)$ correspond to (22).

C.

Let us now show the equivalence between (27) and (29). First, we calculate the integrals shown in (27). We know from [25] that

$$
I_{n l}^{(0)}=\frac{(-1)^{n} 2^{l+2 v+1} l ! \Gamma(l+v+1) \Gamma(n+v+1)}{(l-n) ! n ! \Gamma(n+l+2 v+2)} .
$$

Using the identity $F(a, b ; c ; z)=\Gamma(1-a) \Gamma(c) P_{-a}^{(c-1, a+b-c)}(1-$ $2 z) / \Gamma(c-a)$ we can write

$$
\begin{aligned}
& I_{n l}^{(1)}=\frac{\Gamma\left(1+n^{\prime}\right) \Gamma\left(-2 n^{\prime}-\mu-\nu\right)}{\Gamma\left(-n^{\prime}-\mu-\nu\right)} \int_{-1}^{1} d \vartheta_{0}\left(1-\vartheta_{0}\right)^{\nu+l} \\
& \cdot\left(1+\vartheta_{0}\right)^{\nu} P_{n}^{(v)}\left(\vartheta_{0}\right) P_{n^{\prime}}^{\left(-2 n^{\prime}-\mu-\nu-1, v\right)}\left(\vartheta_{0}\right),
\end{aligned}
$$

where $n^{\prime}=N-l-1$. This integral can be solved by means of (20) of Chapter 16.4 of [28]. We find

$$
\begin{aligned}
I_{n l}^{(1)} & =\frac{2^{2 v+l+1} \Gamma(-l+n) \Gamma(n+v+1) \Gamma(l+v+1)}{n ! \Gamma(-l) \Gamma(2 v+n+l+2)} \\
& \times{ }_{4} F_{3}\left[-n^{\prime},-n^{\prime}-\mu, v+l+1, l+1 ;-2 n^{\prime}-\mu\right. \\
& -v, 2 v+l+n+2,-n+l+1 ; 1]
\end{aligned}
$$

or after using $\Gamma(a-n)=(-1)^{n} \Gamma(-a) \Gamma(1+a) / \Gamma(n+1-a)$

$$
\begin{aligned}
I_{n l}^{(1)} & =(-1)^{n} \frac{2^{2 v+l+1} \Gamma(l+1) \Gamma(n+v+1) \Gamma(l+v+1)}{\Gamma(n-1) \Gamma(1+l-n) \Gamma(2 v+n+l+2)} \\
& \times{ }_{4} F_{3}\left[-n^{\prime},-n^{\prime}-\mu, v+l+1, l+1 ;-2 n^{\prime}-\mu\right. \\
& -v, 2 v+l+n+2,-n+l+1 ; 1] .
\end{aligned}
$$

The remaining integrals can be calculated representing the hypergeometric functions as Meijer $\mathrm{G}$ functions by using the identity

$$
F[a, b, c, z]
$$

$$
=\frac{\Gamma(c)}{\Gamma(b) \Gamma(a)} G_{2} \begin{array}{ll}
1 & 2
\end{array}\left(\begin{array}{cc}
1-a, 1-b \\
0,1-c & \mid-z
\end{array}\right)
$$

and integration identities of the Meijer $G$ functions [29]. Performing the change of variables $-x=\left(1-\vartheta_{1}\right) / 2$ and using the identity $F[a, b ; c ; z]=(1-z)^{c-a-b} F[c-a, c-b ; c ; z][30]$, we get

$$
\begin{aligned}
& J_{k l}^{(0)}=\frac{1}{(-1)^{l+1} 2^{2 \gamma+l}} \int_{0}^{\infty} d x \frac{x^{-\gamma-l-1}}{(x+1)^{\nu}} \\
& \cdot F\left[-v+\frac{1}{2}+i k,-v+\frac{1}{2}-i k ;-v+1 ;-x\right]=\frac{1}{(-1)^{l+1} 2^{2 v+l}} \\
& \cdot \int_{0}^{\infty} d x \frac{F\left[\frac{1}{2}+i k, \frac{1}{2}-i k ;-\nu+1 ;-x\right]}{x^{\nu+l+1}} \\
& =\frac{\Gamma(-v+1)}{(-1)^{l+1} 2^{2 v+l}\left|\Gamma\left(\frac{1}{2}+i k\right)\right|^{2}} \int_{0}^{\infty} d x \\
& \frac{G_{22}^{12}\left(\begin{array}{c}
1 / 2+i k, 1 / 2-i k \\
0, \nu
\end{array} \mid x\right)}{x^{\nu+l+1}}=\frac{\Gamma(-v+1)}{(-1)^{l+1} 2^{2 v+l}|\Gamma(1 / 2+i k)|^{2}}
\end{aligned}
$$

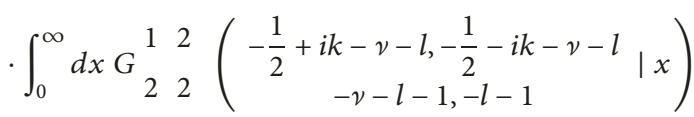

$$
\begin{aligned}
& =\frac{\Gamma(-v+1) \Gamma(-\nu-l)\left|\Gamma\left(\frac{1}{2}+i k+\nu+l\right)\right|^{2}}{(-1)^{l+1} 2^{2 v+l}\left|\Gamma\left(\frac{1}{2}+i k\right)\right|^{2} \Gamma(l+1)} .
\end{aligned}
$$


Finally, similarly to (C.6), we get

$$
\begin{aligned}
& J_{k l}^{(1)}=\frac{1}{(-1)^{l+1} 2^{2 v+l}} \int_{0}^{\infty} d x \frac{x^{-\nu-l-1}}{(x+1)^{\nu}} F\left[-v+\frac{1}{2}+i k,-v+\frac{1}{2}-i k ;-v+1 ;-x\right] \\
& \times F\left[n^{\prime}+1, n^{\prime}+1+\mu ; 2 n^{\prime}+\mu+\nu+2 ;-x\right] \\
& =\frac{\Gamma(-v+1)}{(-1)^{l+1} 2^{2 v+l}\left|\Gamma\left(\frac{1}{2}+i k\right)\right|^{2}} \frac{\Gamma\left(2 n^{\prime}+\mu+\nu+2\right)}{\Gamma\left(n^{\prime}+1\right) \Gamma\left(n^{\prime}+\mu+1\right)}
\end{aligned}
$$

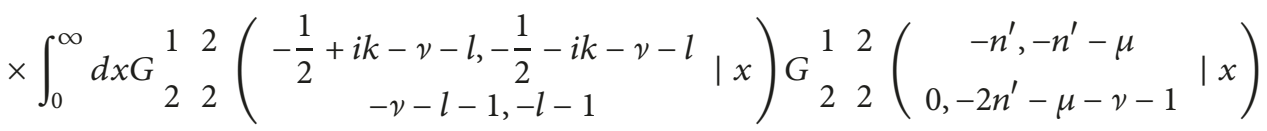

$$
\begin{aligned}
& =\frac{\Gamma(-v+1)}{(-1)^{l+1} 2^{2 v+l}\left|\Gamma\left(\frac{1}{2}+i k\right)\right|^{2}} \frac{\Gamma\left(2 n^{\prime}+\mu+\nu+2\right)}{\Gamma\left(n^{\prime}+1\right) \Gamma\left(n^{\prime}+\mu+1\right)} \\
& \times G_{4}^{3} \quad \begin{array}{ll}
3 \\
{ }_{4}
\end{array}\left(\begin{array}{c}
v+l+1,-n^{\prime},-n^{\prime}-\mu, l+1 \\
\frac{1}{2}-i k+v+l, \frac{1}{2}+i k+v+l, 0,-2 n^{\prime}-\mu-v-1
\end{array}\right) .
\end{aligned}
$$

Once the integrals have been obtained in terms of Meijer $G$ functions, Theorem 1 follows from using the simple identity

$$
\begin{aligned}
& \left(A_{k}^{(-v)}\right)^{2} \sum_{l=0}^{N-1}\left(I_{n l}^{(1)} J_{k l}^{(1)}-I_{n l}^{(0)} J_{k l}^{(0)}\right) \\
& =\frac{2 P_{n}^{(v)}(1)|\Gamma(1 / 2-v+i k)|^{2}}{|\Gamma(i k)|^{2} \varepsilon_{n k}} c_{n k}^{(v)}\left(N_{1}\right) c_{n k}^{(v)}\left(N_{2}\right)
\end{aligned}
$$

or equivalently

$$
\begin{aligned}
& \sum_{l=0}^{N-1}\left(I_{n l}^{(1)} J_{k l}^{(1)}-I_{n l}(0) J_{k l}(0)\right) \\
& \quad=\frac{\Gamma(n+v+1)(\Gamma(-v+1))^{2}}{2^{2 v-1} \Gamma(n+1) \Gamma(\nu+1)} \frac{c_{n k}^{(v)}\left(N_{1}\right) c_{n k}^{(v)}\left(N_{2}\right)}{k^{2}+(n+v+1 / 2)^{2}} .
\end{aligned}
$$

which can be checked with the Meijer $G$ function representation of algebraic computer systems such as Mathematica [31].

\section{Data Availability}

No data were used to support this study.

\section{Conflicts of Interest}

The authors declare that they have no conflicts of interest.

\section{Acknowledgments}

This work was supported by the Brazilian agencies CNPq and CAPES.

\section{References}

[1] C. W. J. Beenakker, "Random-matrix theory of quantum transport," Reviews of Modern Physics, vol. 69, no. 3, pp. 731-808, 1997.

[2] A. Altland and M. R. Zirnbauer, "Nonstandard symmetry classes in mesoscopic normal-superconducting hybrid structures," Physical Review B: Condensed Matter and Materials Physics, vol. 55, no. 2, pp. 1142-1161, 1997.

[3] M. Caselle, "A new classification scheme for random matrix theories," 1996, https://arxiv.org/abs/cond-mat/9610017.

[4] P. W. Brouwer and A. Altland, "Anderson localization from classical trajectories," Physical Review B: Condensed Matter and Materials Physics, vol. 78, no. 7, Article ID 075304, 2008.

[5] K. Efetov, Supersymmetry in Disorder and Chaos, Cambridge University Press, Cambridge, UK, 1997.

[6] K. Richter and M. Sieber, "Semiclassical theory of chaotic quantum transport," Physical Review Letters, vol. 89, article 206801, 2002.

[7] G. Berkolaiko and J. Kuipers, "Universality in chaotic quantum transport: the concordance between random-matrix and semiclassical theories," Physical Review E: Statistical, Nonlinear, and Soft Matter Physics, vol. 85, article 045201, 2012.

[8] S. R. Elliott and M. Franz, "Colloquium: Majorana fermions in nuclear, particle, and solid-state physics," Reviews of Modern Physics, vol. 87, no. 1, pp. 137-163, 2015.

[9] J. P Dahlhaus, Random-matrix theory and stroboscopic models of topological insulators and superconductors, Lorentz Institute, Faculty of Science, Leiden University, Leiden, The Netherlands, 2012.

[10] C. W. Beenakker, "Random-matrix theory of Majorana fermions and topological superconductors," Reviews of Modern Physics, vol. 87, no. 3, pp. 1037-1066, 2015.

[11] P. W. Brouwer, A. Furusaki, I. A. Gruzberg, and C. Mudry, "Localization and delocalization in dirty superconducting 
wires," Physical Review Letters, vol. 85, no. 5, pp. 1064-1067, 2000.

[12] F. J. Dyson, "The dynamics of a disordered linear chain," Physical Review A: Atomic, Molecular and Optical Physics, vol. 92, no. 6, pp. 1331-1338, 1953.

[13] O. Motrunich, K. Damle, and D. A. Huse, "Griffiths effects and quantum critical points in dirty superconductors without spin-rotation invariance: one-dimensional examples," Physical Review B: Condensed Matter and Materials Physics, vol. 63, no. 22, article 224204, 2001.

[14] I. A. Gruzberg, N. Read, and S. Vishveshwara, "Localization in disordered superconducting wires with broken spin-rotation symmetry," Physical Review B: Condensed Matter and Materials Physics, vol. 71, no. 24, article 245124, 2005.

[15] C. Mudry, "Fractional abelian topological phases of matter for fermions in two-dimensional space," Topological Aspects of Condensed Matter Physics, vol. 103, article 265, 2017.

[16] A. F. Macedo-Junior and A. M. Macêdo, "Brownian-motion ensembles of random matrix theory: a classification scheme and an integral transform method," Nuclear Physics, vol. 752, no. 3, pp. 439-475, 2006.

[17] A. F. Macedo-Junior, Transporte Em Nanoestruturas: Métodos De Movimento Browniano E Teoria De Circuitos, Universidade Federal de Pernambuco, 2006.

[18] S. Datta, Electronic Transport in Mesoscopic Systems, Cambridge University Press, Cambridge, UK, 1997.

[19] J. P. Dahlhaus, B. Béri, and C. W. Beenakker, "Random-matrix theory of thermal conduction in superconducting quantum dots," Physical Review B: Condensed Matter and Materials Physics, vol. 82, no. 1, Article ID 014536, 2010.

[20] M. I. Sena-Junior, F. A. Almeida, and A. M. Macêdo, "Counting statistics and an anomalous metallic phase in a network of quantum dots," Journal of Physics A, vol. 47, Article ID 235101, 2014.

[21] S. Iida, H. A. Weidenmüller, and J. A. Zuk, "Statistical scattering theory, the supersymmetry method and universal conductance fluctuations," Annals of Physics, vol. 200, no. 2, pp. 219-270, 1990.

[22] A. Macêdo, "Quantum dot to disordered wire crossover: A complete solution in all length scales for systems with unitary symmetry," Physical Review B: Condensed Matter and Materials Physics, vol. 61, no. 7, pp. 4453-4456, 2000.

[23] G. C. Duarte-Filho, A. F. Macedo-Junior, and A. M. Macêdo, "Circuit theory and full counting statistics of charge transfer through mesoscopic systems: A random-matrix approach," Physical Review B: Condensed Matter and Materials Physics, vol. 76, no. 7, Article ID 075342, 2007.

[24] C. E. Souza and A. F. Macedo-Junior, "Quantum transport in chaotic ballistic cavity: a Brownian-motion approach," Journal of Physics A: Mathematical and General, vol. 48, no. 41, Article ID 415101, 2015.

[25] A. F. Macedo-Junior and A. M. Macêdo, "Brownian-motion ensembles: correlation functions of determinantal processes," Journal of Physics A: Mathematical and General, vol. 41, no. 1, Article ID 015004, 2008.

[26] A. M. Macêdo, "Nonanalytic scaling of conductance cumulants in dirty superconducting wires," Physical Review B: Condensed Matter and Materials Physics, vol. 65, no. 13, Article ID 132510, 2002.

[27] A. D. Mirlin, A. Muller-Groeling, and M. R. Zirnbauer, "Conductance fluctuations of disordered wires: Fourier analysis on supersymmetric spaces," Annals of Physics, vol. 236, no. 2, pp. 325-373, 1994.

[28] H. Bateman and A. Erdelyi, Higher Transcendental Functions, vol. 2, McGraw-Hill, New York, NY, USA, 1953.

[29] I. S. Gradshteyn and I. M. Ryzhik, Table of Integrals, Series, and Products, Academic Press, 2014.

[30] F. W. J. Olver, NIST Handbook of Mathematical Functions Hardback and CD-ROM, Cambridge University Press, Cambridge, UK, 2010.

[31] Wolfram Research, Inc., Mathematica, Version 10.2, Champaign, IL, 2017. 


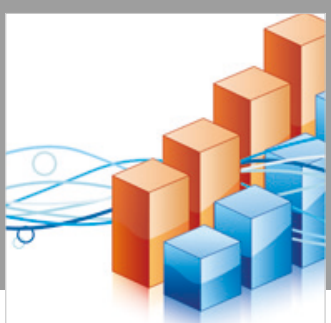

Advances in

Operations Research

\section{-n-m}
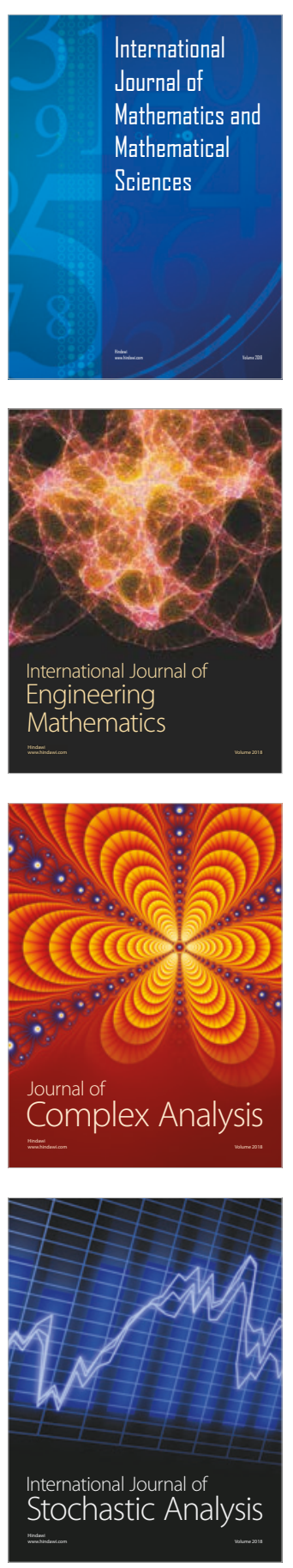
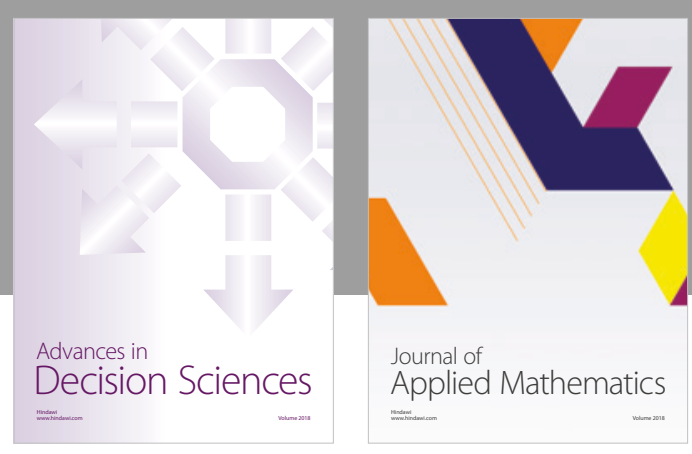

Journal of

Applied Mathematics
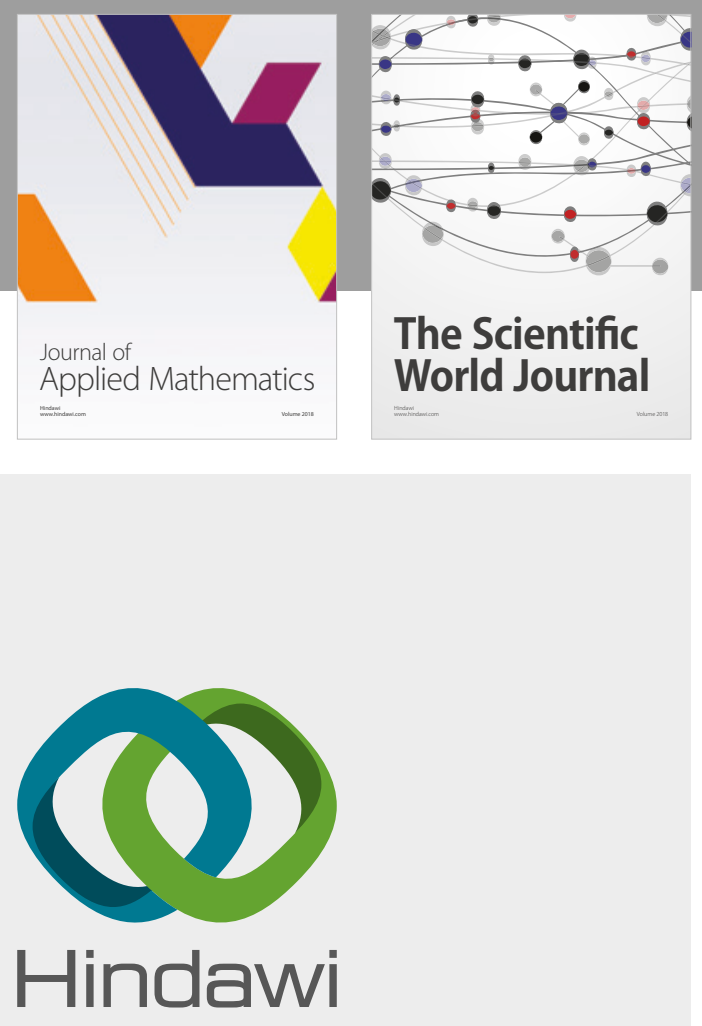

Submit your manuscripts at

www.hindawi.com

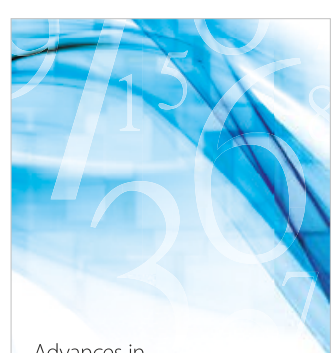

Advances in
Numerical Analysis
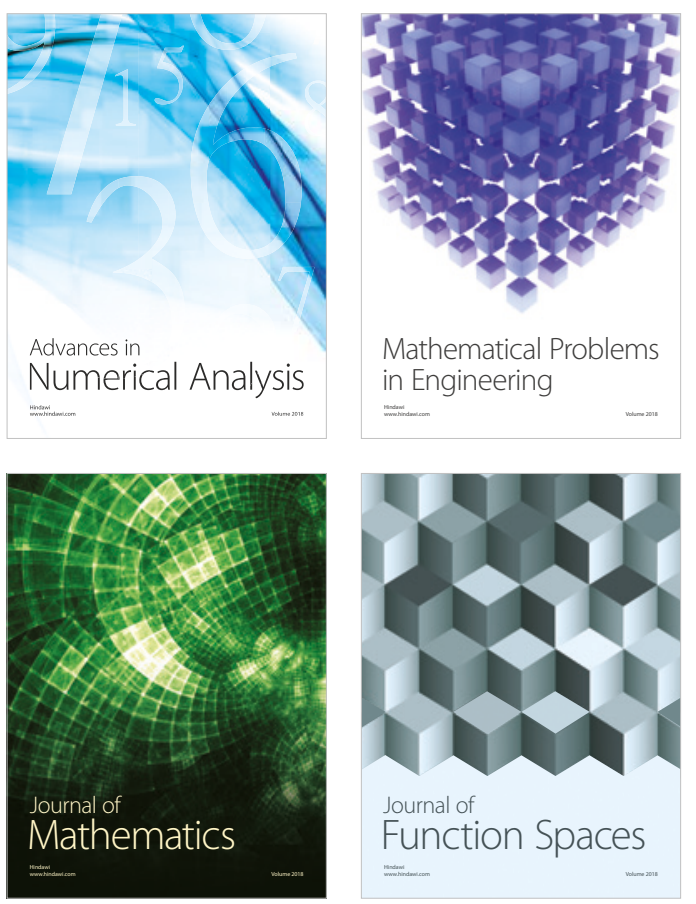

Mathematical Problems in Engineering

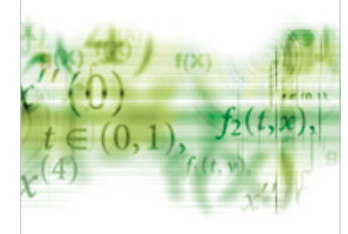

International Journal of

Differential Equations

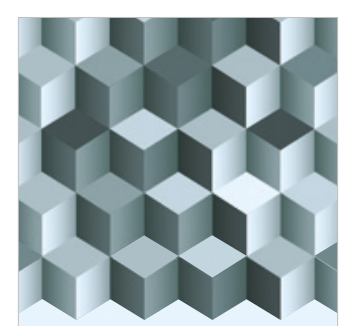

Journal of

Function Spaces

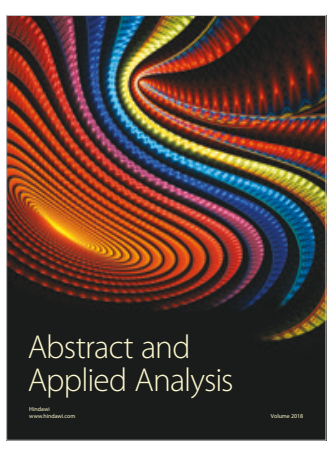

The Scientific

World Journal

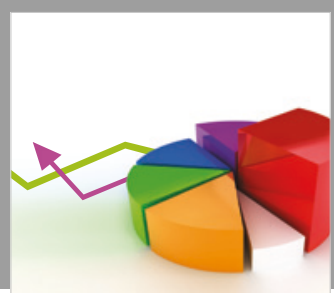

Journal of

Probability and Statistics
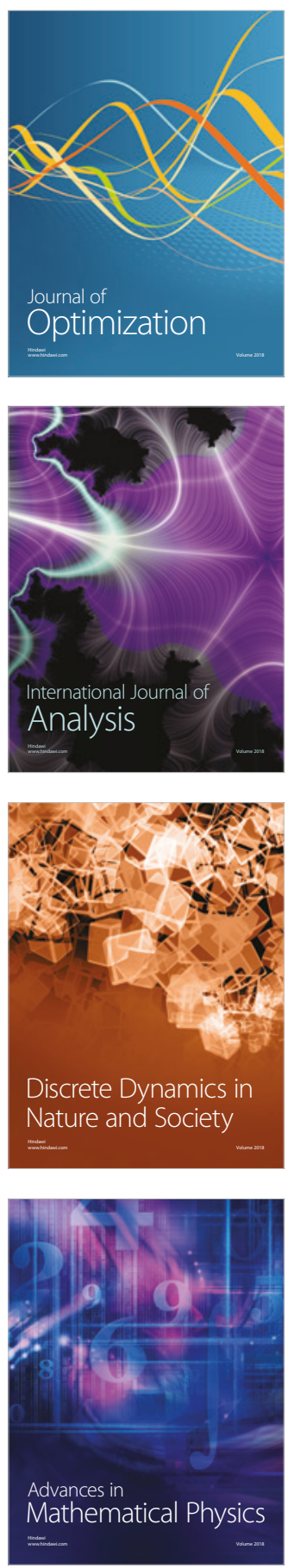